\title{
You Gotta Walk the Walk to Talk the Talk: Happy Older Latino Adults (HOLA) as a pilot health promotion and prevention program for older HIV-positive Latino men
}

Daniel Jimenez ( $\nabla$ dej18@miami.edu )

University of Miami School of Medicine https://orcid.org/0000-0003-2135-0619

Elliott R. Weinstein

University of Miami - Coral Gables Campus: University of Miami

John Batsis

University of North Carolina at Chapel Hill School of Medicine

Methodology

Keywords: Latinos, older adults, health promotion, HIV, cardiometabolic risk

Posted Date: April 2nd, 2021

DOI: https://doi.org/10.21203/rs.3.rs-348183/v1

License: (-) (i) This work is licensed under a Creative Commons Attribution 4.0 International License. Read Full License 


\section{Abstract \\ Background}

Older Latinos with HIV have been disproportionately affected by the epidemic and experience compounded health disparities that have deepened over time. These health disparities are largely related to lifestyle and are either preventable or amenable to early detection or intervention. Despite existing resources to deliver an intervention to reduce this compounded health disparity, there is little information on the effects of health promotion interventions on indices of cardiometabolic risk in midlife and older Latinos with HIV. The Happy Older Latinos are Active (HOLA) intervention is an innovative health promotion program that is uniquely tailored to meet the diverse needs and circumstances of older Latinos with HIV. The goal of this pilot study is to evaluate the feasibility and acceptability of HOLA among Latinos aged 50 and older with HIV.

\section{Methods/Design:}

HOLA, which is informed by Behavioral Activation and Social Learning theory is a community health worker (CHW)-led, multicomponent, health promotion intervention consisting of: (1) a social and physical activation session; (2) a moderately intense group walk led by a CHW for 45 minutes, $3 x /$ week for 16 weeks; (3) pleasant events scheduling. The proposed pilot study will recruit and enroll 18 community dwelling Latinos with HIV aged 50+. Participants will be assessed at three time points (baseline, post intervention, and 3 months post intervention) on measures of cardiometabolic risk factors (waist circumference, dyslipidemia, hypertension, and glucose), psychosocial functioning, and health-related quality of life.

\section{Conclusions}

If HOLA can be delivered successfully by CHWs, then the scalability, accessibility, and potential for dissemination is increased. Additionally, the results of this pilot study have far reaching implications in regard to the health and welfare of other at-risk, under resourced populations. This pilot study will inform feasibility and identify modifications needed in the design of a larger hypothesis testing study.

\section{Background}

Due to the changing landscape of HIV, older adults are living with HIV at rates higher than ever before. In 2018, the prevalence of diagnosed HIV infection in the United States (U.S.) was 374.6 per 100,000 population with an increased number of estimated cases affecting older Americans over the age of $50 .^{1}$ Even though HIV is no longer a death sentence due to the development of effective antiretroviral therapy (ART) and other HIV focused therapeutics, significant disparities in treatment outcomes persist among certain demographics. In general, HIV continues to not only affect racial/ethnic minorities at higher rates, but also gay, bisexual, and men who have sex with men (MSM) compared to their cis-gender heterosexual white counterparts. ${ }^{2}$ Therefore, research must look to the intersections of age, race, ethnicity, and sexual identity in developing interventions that may best support those most at risk for poor HIV-related treatment outcomes.

Significantly more people with HIV (PWH) are living into older adulthood demonstrating a growing need for HIV-focused research and treatment examined through an aging lens. According to 2018 CDC data, more than half of PWH in the U.S. are over the age of 50 years ${ }^{1}$ with predictions that by the start of 2021 almost $70 \%$ of PWH in the United States will be at least 50 years old. ${ }^{3}$ Older adults are often diagnosed with HIV much later ( 4.5 years after infection) indicating more advanced disease progression associated with the epidemic among this age group. ${ }^{1}$ Additionally, for PWH in the United States aged 55 and above, while $90 \%$ knew their HIV status, only $64 \%$ were virally suppressed and barely more than half (57\%) were actively engaged in care, suggesting that the "90-90-90" goals towards ending the HIV epidemic ${ }^{4}$ are currently out of reach among older adults with HIV (OAWH). ${ }^{1}$

Latinos have been disproportionately affected by HIV since the origins of the epidemic, and that increased vulnerability has deepened over time. Despite representing less than $20 \%$ of the entire U.S. population, Latinos make up $27 \%$ of new HIV diagnoses and account for over $20 \%$ of the national prevalence. ${ }^{5}$ More specifically, Latino sexual minority men (LSMM) are responsible for almost $25 \%$ of new HIV infections among all gay, bisexual, and MSM identified individuals ${ }^{2}$ despite Latino men making up less than $9 \%$ of the entire U.S. population. ${ }^{6}$ These disparities persist beyond just HIV incidence with Latino identified PWH facing additional challenges with their HIV treatment care due to suboptimal rates of viral suppression ${ }^{7,8}$ and ART adherence ${ }^{9}$ compared to their non-Latino SMM peers.

Unsurprisingly, these racial/ethnic disparities become significantly more observable among older adults. Older Latinos in the U.S. are slightly more than 5 times more likely to acquire $\mathrm{HIV}^{10}$ and are more likely to have an AIDS diagnosis, detectable viral load, and poorer treatment adherence compared to their age-matched white non-Latino counterparts. ${ }^{11,12}$ Additionally, older Latinos seem to exhibit mild to moderate cognitive impairment in learning, memory, and processing speed compared to their non-Latino white peers. ${ }^{12,13}$ It is likely that these intersecting social determinants (i.e. age, race/ethnicity, sexual identity) will continue as the HIV population ages and that many of these poor health outcomes will be felt most intensely by older Latinos.

Older Latinos with HIV experience various physical health disparities due to their intersecting minority status that must be considered. In general, OAWH face increased rates of age-related comorbidities due to a phenomenon called accelerated aging. ${ }^{14,15}$ Data from a large observational cohort study of OAWH reported that over $77 \%$ of participants reported suffering from two or more health comorbidities in addition to HIV ${ }^{16}$ with the average number of physical comorbidities per participants landing at three. ${ }^{17}$ In particular, older HIV-positive Latino individuals are disproportionately affected with cardiometabolic diseases including metabolic syndrome (MetS), a precursor to diabetes, as well as cardiometabolic risk factors such as obesity and hypertension compared

Page 2/11 
to their non-Latino older white peers. ${ }^{18,19,20}$ Moreover, older Latinos with HIV are more likely to be sedentary and not as actively engaged in pursuing changes in their physical activity compared to their non-Latino white counterparts. ${ }^{21}$ Despite evidence of the "Hispanic Paradox" 22 , this lack of physical activity in combination with possible issues connected to ART medication and Hepatitis-C co-infection make older Latino adults with HIV more likely to suffer from other complicating physical conditions like nonalcoholic fatty liver disease $\mathrm{e}^{23,24}$ and cardiovascular issues compared to their non-Latino white counterparts.

Older Latinos with HIV face elevated rates of mental health concerns in addition to these physical comorbidities. More generally, OAWH have documented rates of major depressive episodes anywhere between 18 and $52 \%{ }^{17,26,27}$, significantly higher than the $1-8 \%$ documented rates of depression among older adults in the general population. ${ }^{28}$ Rates of social isolation in the general aging public (60 years or older) are estimated to be anywhere between $33 \%$ and $50 \%{ }^{29}$ with evidence suggesting that OAWH face social isolation more often than their age matched peers in the general population. ${ }^{30,31}$ Similarly, there is strong evidence to suggest that loneliness exponentially increases with age and one could predict OAWH bear even a greater amount of loneliness due to reduced social networks and ostracism. ${ }^{32,33}$ Additionally, loneliness and social isolation have been correlated with levels of morbidity and mortality comparable to more established biopsychosocial risk factors like obesity, sedentary behavior, smoking, and hypertension. ${ }^{29,34,35}$

For OAWH, one of the biggest contributing factors to loneliness and social isolation is that of co-occurring stigma. While exact estimates of HIV stigma and age-related stigma in the U.S. are hard to calculate, OAWH must often navigate the dueling stigmas of HIV stigma, ageism, and stigma resulting from other possible marginalized identities. ${ }^{36-38}$ It is possible that rates of stigma and loneliness seem to skyrocket in OAWH due to the increased likelihood that OAWH live along and have limited and often inconsistent social networks. ${ }^{26,38} \mathrm{OAWH}$ can face ostracism from the larger LGBTQ + community and stigma due to the intersection of their age and HIV status compared to their non-infected peers which may in turn contribute to the increased levels of depression among this already vulnerable population. ${ }^{39}$ Additionally, since familism and social cohesion are strong hallmarks of Latino culture, ${ }^{40,41}$ Latino $0 A W H$ may experience the harmful effects of co-morbid stigma and social isolation more intensely than their non-Latino HIV-positive peers due to societal expectation that they be more connected to family as they age; however, more research must be conducted to determine the veracity of such a hypothesis.

As outlined above, compounded health disparities place older Latinos with HIV at particularly high risk for diminished quality of life due to physical and mental health morbidity. These data underscore the public health importance of increased efforts to address the multiplicative and unequal burden of HIV, MetS, and diabetes shouldered by older Latinos. Therefore, based on this gap in the literature, there is a compelling need to develop and disseminate interventions that promote healthy living, combat social isolation, and improve HIV-related health outcomes among older Latinos with HIV. Since this is a pilot study, researchers are focused on establishing feasibility and acceptability of a potential intervention rather than testing specific hypotheses. Also, in accordance with recommendations from biostatistical workgroups funded by $\mathrm{NIH}^{42}$ this pilot study is not powered to test a hypothesis. Rather, this pilot study serves as an initial step in establishing feasibility and acceptability of an innovative application of an already established health promotion intervention titled HOLA. ${ }^{43}$ The specific aims of this pilot study are:

1. To evaluate the feasibility of recruitment, assessment procedures, retention, acceptability, and implementation of HOLA in a sample of midlife and older Latinos with HIV.

2. To identify modifications needed in the design of a larger, confirmatory randomized controlled trial.

3. To explore changes in cardiometabolic risk factors (waist circumference, dyslipidemia, hypertension, and glucose), psychosocial functioning (depression and anxiety severity, social support), and health-related quality of life in a sample of midlife and older Latinos with HIV enrolled in the HOLA health promotion intervention.

\section{Methods}

All study methods and protocols were approved by the university's Internal Review Board (IRB ID: 20181032).

\section{Participants}

This pilot study will enroll 18 Latino older (aged 50+) men with HIV to three specific cohorts of the HOLA intervention. Despite the small sample size, 18 participants should be large enough to observe meaningful differences in primary outcomes of interest and establish feasibility of the intervention in a population of older Latino men with HIV. Additionally, this sample size is consistent with similar pilot studies focused on physical activity among older adults. ${ }^{44,45}$ Informed consent was obtained from each participant prior to enrollment and all enrollees were provided with an initial verbal summary of the study. Payments were graduated so participants received $\$ 15$ on the first visit, $\$ 25$ on the second visit, and $\$ 35$ on the third visit (total of honoraria $=\$ 75$ ). For a detailed description of the inclusion/exclusion criteria, see Table 1. 
Table 1

Inclusion/Exclusion Criteria

\begin{tabular}{|c|c|}
\hline $\begin{array}{l}\text { Inclusion } \\
\text { Criteria }\end{array}$ & Exclusion Criteria \\
\hline $\begin{array}{l}\text { Latino (self- } \\
\text { identified) }\end{array}$ & Have a diabetes diagnosis \\
\hline Age 50+ & $\begin{array}{l}\text { Have a diagnosis of any neurodegenerative disorder or dementia (Parkinson's disease, Alzheimer's, vascular, frontotemporal } \\
\text { dementia, etc.) or significant cognitive impairment as indicated by a Mini Mental Status Exam score }<24\end{array}$ \\
\hline Male & Have contraindications to physical activity outlined in the American College of Sports Medicine standards \\
\hline $\begin{array}{l}\text { HIV infected but } \\
\text { are virologically } \\
\text { suppressed } \\
\text { (viral load < } 200 \\
\text { copies } / \mathrm{mL} \text { ) }\end{array}$ & Are unable to complete $10 \mathrm{~m}$ walk test \\
\hline $\begin{array}{l}\text { Volunteer } \\
\text { informed } \\
\text { consent }\end{array}$ & Currently residing in a nursing or group home \\
\hline $\begin{array}{l}\text { Expect to stay } \\
\text { in Miami for the } \\
\text { next } 6 \text { months }\end{array}$ & Have a terminal physical illness expected to result in the death within one year \\
\hline \multirow[t]{2}{*}{$\begin{array}{l}\text { Have } \\
\text { documented } \\
\text { risk of } \\
\text { cardiometabolic } \\
\text { disease. }\end{array}$} & $\begin{array}{l}\text { Have an acute or severe medical illness that precludes them from safely participating in a health promotion intervention (e.g. } \\
\text { progressive, degenerative neurologic disease, such as Parkinson's Disease, multiple sclerosis, ALS; severe arthritis or orthopedic } \\
\text { condition that would prevent participation in a physical activity program; lung disease requiring either oral or injected steroids, or the } \\
\text { use of supplemental oxygen; New York Heart Association Class III or IV congestive heart failure, clinically significant aortic stenosis, } \\
\text { history of cardiac arrest, use of a cardiac defibrillator, or uncontrolled angina; renal disease requiring the use of dialysis; cancer being } \\
\text { actively treated with radiation or chemotherapy; myocardial infarction, CABG, or valve replacement within the past } 6 \text { months; serious } \\
\text { conduction disorder, such as 3rd degree heart block; uncontrolled arrhythmia; pulmonary embolism or deep venous thrombosis within } \\
\text { past 6-months; uncontrolled diabetes with recent weight loss, diabetic coma or frequent insulin reactions; stroke, hip fracture, hip or } \\
\text { knee replacement, or spinal surgery in the past } 6 \text { months; receiving physical therapy for gait, balance, or other lower extremity } \\
\text { training; severe, uncontrolled hypertension -systolic blood pressure }>200 \text { mmHg and/or diastolic blood pressure }>110 \text { mmHg) }\end{array}$ \\
\hline & Are currently taking antidepressant medications in doses indicated for weight reduction. \\
\hline
\end{tabular}

\section{Recruitment}

Participants will be recruited through two consent-to-contact databases with over 1200 participants each - one of people with HIV (recruited from the university-affiliated adult HIV clinic) and another of people at risk for acquiring HIV (recruited from the community). Data in these databases include contact information, demographic information and data associated with HIV-related risk factors such as homelessness and psychological distress.

\section{Intervention}

Happy Older Latino Adults (HOLA) is a multi-component health promotion intervention for midlife and older Latinos. ${ }^{43}$ The conceptual model which serves as the foundation for the intervention (Fig. 1) was crafted to address comorbid depression and anxiety symptoms as well as both physical and psychosocial functioning of older Latinos. Grounded in theory, HOLA addresses the goals mentioned above through two interconnected mechanisms, Social Learning Theory (SLT) ${ }^{46}$ and Behavioral Activation (BA) ${ }^{47} \mathrm{BA}$ is used to engage participants in both a physical activity routine and pleasant events scheduling to combat incident and recurrent episodes of depression and anxiety disorders as well as subdue symptom intensity. ${ }^{47}$ As a complement, SLT's tenets of reinforcement, observational learning, and enhanced self-efficacy are utilized to bolster participant engagement and success in the intervention. ${ }^{46}$ Additionally, using a task-shifting approach the intervention is delivered by a community health worker (CHW) who capitalizes on the unique relationship they have with participants to model, encourage, and promote consistent health behavior change. ${ }^{48,49}$ Accountability is established in two ways - between an individual and the $\mathrm{CHW}$ as well as an individual and the larger group - in an effort to cultivate extra engagement and motivation in the intervention.

The first component of this iteration of HOLA are two structured social and physical activation sessions. Each enrolled participant first meets with the CHW for an individualized 30-minute physical and social activation session in anticipation for the group exercise phase of the intervention. This session serves five purposes - (a) to educate potential participants about the overall goals of the program; (b) inspire participants to take part in physical activity, (c) increase the sheer number of participants' social activities; (d) recognize possible challenges that may prevent participants' from successfully engaging with the intervention; and (e) problem solve ways to overcome these obstacles. After week 8, participants again meet one-on-one with the CHW for the second session so that they may discuss their own individual progress in relation to their physical and social activity goals.

The second core tenet of HOLA is centered around a group walk meant to facilitate both physical activity and social interaction between participants. This moderately intense group walk meets for 45 minutes, three times a week, for a total of 16 weeks. Moderate intensity is defined as a rating between 11 and 13 on the Borg Scale of Perceived Exertion. ${ }^{50}$ The group walk component was designed with interval training in mind and gradually increases in workload (defined by intensity, volume, and work/recovery cycle) over the course of the intervention. Each group walk begins with 10 minutes of stretching and warm up, followed by 30 minutes of walking, and ends with 5 minutes of stretching/cool down. Each group walk is led by the CHW and is composed of six bilingual and monolingual Spanish-speaking participants (one cohort).

A third component of HOLA centers on scheduling pleasant events in participants' daily routines. In addition to stretching, the cool down phase will feature a quick discussion led by the $\mathrm{CHW}$ in which they ask each participant to identify a pleasant event that they intend to do with another person prior to the next

Page 4/11 
walk session. Activities mentioned may be done alongside other members in the group, or in conjunction with family and friends outside the intervention. The $\mathrm{CHW}$ will be adept at utilizing problem solving skills (i.e. brainstorming) to assist participants should they struggle to commit to a pleasant event. Each subsequent session will begin with a quick recap where participants report back to the group on how successful they were in executing their pleasant event plan while their peers and $\mathrm{CHW}$ provide encouragement, positive feedback, and constructive feedback. Overall, this component is meant to not only generalize the intervention into the participants' everyday lives and relationships, but also to provide positive feedback so that participants can build selfefficacy as they engage with the program.

The fourth and final component of HOLA in the context of this pilot study centers around a maintenance phase to reinforce behavior change gleaned during intervention. Participants will engage in "booster" walking sessions, twice a month for three months post intervention so to capitalize on beneficial physical and mental health effects gained during the 16-week program. Encouraged by the prior literature, this maintenance phase was added to this pilot study with the hopes of cultivating more sustained treatment effects over time. ${ }^{51-53}$

\section{COVID-19 Adaptations}

Due to COVID-19, the protocol has been amended to conduct all intervention-related activities over the telephone. Participants would conference call with the $\mathrm{CHW}$ and the other members of their group at the time of their regularly scheduled walk, and they would walk around their respective neighborhoods with the CHW leading them.

\section{Measures and Analysis}

This quasi-experimental pilot study of a health promotion intervention will primarily examine feasibility of recruitment, assessment procedures, retention, acceptability, and implementation of HOLA in a sample of older Latinos with HIV. In keeping with guidance from NIH funded biostatistical workgroups, this pilot study was not designed to be powered to test a hypothesis. ${ }^{42}$ Additionally, the proposed analysis of feasibility data parallels a similar structure employed in the first HOLA trial and comparable pilot trials. ${ }^{54,55}$ For this study, recruitment was defined as a success should $100 \%$ percent of the targeted sample $(N=18)$ be enrolled and less than $20 \%$ of eligible subjects refusing to participate. Additionally, adequate retention was characterized as $85 \%$ or more of enrolled participants completing all post-intervention assessments while acceptability was defined as subjects' attending at least $80 \%$ of sessions. Finally, with goals of scaling up the intervention in the future, an established project evaluation questionnaire developed by investigators at the University of Miami will be used to pinpoint any modifications needed for the design of a larger, confirmatory randomized control trial. The questionnaire is made up of a series of closed ended yes/no questions, rating scales, and open-ended questions that allow for more qualitative data regarding participants' opinions of the specific components of the overall intervention. Study measures will be administered at baseline, end of intervention, and 3 months post intervention. Trained research assistants (RA) will administer all of the assessments.

\section{Study Measures}

In the full-scale trial, the primary outcomes of this study will be changes in (a) cardiometabolic risk factors as defined by dyslipidemia, glucose level, hypertension, and waist circumference; (b) psychosocial functioning as characterized by depression severity, anxiety severity, and social support; and (c) health related quality of life. A three-pronged screening process was conducted to identify eligible participants. Based on our exclusion criteria, participants completed the Mini Mental Status Exam (MMSE) to recognize potential subjects with dementia or severe cognitive abnormalities. ${ }^{56}$ Second, to establish baseline walking ability, eligible participants were required to complete the 10-meter walk test which has been shown to have not only excellent reliability when used in older adult populations, but also be comparable in validity with other longer measures. ${ }^{57}$ Participants' viral load was ascertained to determine viral suppression which is defined as $<200$ copies $/ \mathrm{mL} .{ }^{58}$ Finally, potential participants self-confirmed safe participation by not having an acute or severe medical illness.

\section{Cardiometabolic Measurements}

Just prior to baseline, blood draws were completed at the university affiliated adult HIV outpatient clinic from which participant recruitment will take place. These blood draws yielded baseline data on participants' levels of HDL-C, LDL-C, triglycerides, insulin, and HbA1c. Fasting blood samples were drawn via venipuncture and stored at $4^{\circ} \mathrm{C}$ until analysis could be completed. Insulin resistance was defined by calculating a participants' homeostatic model assessment (HOMA) score. All the samples were evaluated by commercial laboratory services using commercially available enzyme-linked immunosorbent assays. Simultaneously, participant physical characteristics such as blood pressure and hip-to-waist circumference will be collected to complement the measures of glucose, insulin resistance, and blood lipid profile mentioned prior. All of these measurements of cardiometabolic risk will be collected immediately post intervention, and 3 months post-intervention as well.

\section{Psychosocial Functioning}

Measures of psychosocial functioning will be collected at all three timepoints as well -baseline, post-intervention, and three-months post intervention. To measure depression symptom severity, participants will complete the Center for Epidemiologic Scale of Depression ${ }^{59}$, nine item Patient Health Questionnaire ${ }^{60}$, the Perceived Stress Scale ${ }^{61}$, and the seven-item Generalized Anxiety Disorder scale to ascertain anxiety symptom intensity. ${ }^{62}$ Finally, participants' perceived social support will be measured by the frequently used and validated Multidimensional Scale of Perceived Social Support. ${ }^{63}$

\section{Additional Measures}

Additional measures will be administered to participants to gain more insight on factors of acculturation, physical activity, stigma, quality of life, and overall patient satisfaction. At baseline, participants will complete a demographics form, provide a list of current medications, and respond to the Bidimensional Acculturation Scale. ${ }^{64}$ Additionally, at all three time points - baseline, post-intervention, and three-months post intervention - subjects will be asked to 
complete the Global Physical Activity Questionnaire, ${ }^{65}$ the 12 -item Short Form health survey, ${ }^{66}$ and the HIV Stigma Scale ${ }^{67}$ to measure their overall physical activity levels, general health-related quality of life, and HIV-related stigma participants face on a day-to-day basis. Finally, participants will complete a project evaluation questionnaire developed by the investigators to indicate individual satisfaction in the intervention.

\section{Analysis}

Before analyzing the pilot data collected, all electronic data will be stripped of identifiers and will be stored in password-protected files. Specifically, for each data file, a code number will be assigned, and the master list, linking code numbers with names, will be stored separately. As per IRB protocol, collected data will be stored on password protected, network computer hard drives, which will only be accessible to the Principal Investigator to maintain patient privacy and confidentiality.

Overall, the purpose of this pilot study is not to test hypotheses, but rather generate them. The main goal of this study is to explore possible hypotheses concerning the intervention's effects on cardiometabolic risk factors, psychosocial functioning, and health-related quality of life in a sample of older Latinos with HIV. After data collection, all data will be exported to SPSS for analysis. A one-way ANOVA will be used to illustrate the change in scores from baseline to post-intervention in all the outcome measures used. Since this is a pilot study without a control group, a repeated measures ANOVA is appropriate to explore the intervention effects on cardiometabolic risk factors, psychosocial functioning, and health-related quality of life given that this type of analysis is often used in studies that investigate either changes in mean scores over three or more time points. ${ }^{68}$ For exploratory analyses, we will incorporate a number of covariates including age, socioeconomic status, and years of education. Similarly, changes in current medications or dosage levels will be controlled for during the exploratory analysis. Methodologically, covariates will be tested as moderators of treatment response.

\section{Discussion}

As the population of PWH increasingly ages, high comorbidity between HIV and cardiometabolic diseases make older Latinos a high-risk population for whom innovative, scalable health promotion intervention could make a significant and lasting public health impact. ${ }^{19}$ Despite the fact that older Latinos with HIV experience high levels of cardiometabolic disease due to issues associated with accelerated aging or potential adverse effects of ART medication, there is still a considerable dearth of relevant information on the effects of positive health promotion interventions in older Latinos living with HIV and comorbid cardiometabolic illness. This study will generate evidence as to whether or not an already established health promotion intervention, HOLA, can be adapted for older Latino men with HIV to improve physical health, psychosocial functioning, and health-related quality of life in the midst of a rapid demographic transition.

The HOLA intervention is a multifaceted, innovative health promotion program that is uniquely tailored to meet the diverse needs and circumstances of older Latinos with HIV. Since many older adults who identify as racial/ethnic minorities hold stigmatizing views of mental health services, ${ }^{69,70} \mathrm{HOLA}$ builds on prior research and incorporates culturally relevant strategies to health promotion/behavior change as way of reducing mental and physical health risk factors among older Latinos with HIV. ${ }^{71}$ Specifically, the use of a CHW to deliver a dual mental/physical health promotion intervention is an innovative approach to minimize disease burden in a population with high exposure to risk factors (in addition to HIV) and established disparities in both access to and engagement in beneficial mental and physical health services. ${ }^{70,72,73}$ We believe that such an approach will appeal to older Latinos with HIV at risk for cardiometabolic disorders and psychological distress because of its non-stigmatizing presentation and the incorporation of cultural values/beliefs that promote the varying sociocultural influences contributing to the health of Latinos. Essentially, if HOLA can be delivered successfully by CHWs, then the scalability, accessibility, and potential for dissemination is increased and HOLA becomes an optimal choice to address a significant medical and public health issue.

\section{Conclusions}

To our knowledge, HOLA is the first health promotion trial whose central focus is on improving both physical and mental health among older Latinos with HIV. As the literature suggests, increased physical activity and the incorporation of pleasant events into daily routine are both effective strategies in treating geriatric anxiety and depression.47,74.75 Therefore, the integrated (mental and physical health) nature of this health promotion intervention bolsters its novelty and potential clinical impact. Additionally, the results of this pilot study have far reaching implications in regard to the health and welfare or other atrisk, under resourced populations. Since, the intervention is culturally tailored and contains features that will enable easy adaptation to different Latino subgroups, there's potential for further modification to other disadvantaged groups like older African-Americans with HIV or older sexual minorities with HIV. Like older Latino adults with HIV, older African-Americans with HIV report low utilization of mental health services, 76 high rates of physical and mental health comorbidities,77 and significant experiences of ageism and HIV related stigma78 demonstrating just how impactful a program like HOLA may be when implemented within the community. Overall, the dissemination of a health promotion intervention like HOLA in a population of older Latino adults with HIV can serve to tackle multiple disparities (both health and service utilization related) among vulnerable adults at the crux of multiple marginalized communities (racial/ethnic, age, and HIV-status).

\section{Declarations}

\section{Ethics approval and consent to participate}

All study methods and protocols were approved by the University of Miami's Internal Review Board (IRB ID: 20181032). 
Not applicable

Availability of data and materials

Not applicable

Competing interests

The authors declare that they have no competing interests.

Funding

This research was supported by grants R01 MD012610 and U54 MD002266 from the National Institute on Minority Health and Health Disparities and R01 AG053163 from the National Institute on Aging. Dr. Batsis is supported by K23-AG051681 from the National Institute on Aging.

Authors' contributions

DJ is the study principal investigator and contributed to the theoretical background and conceptualization of the study, intervention and study design, writing, and editing. EW and JB contributed to writing and editing. All authors read and approved the final manuscript.

Acknowledgements

Not applicable

\section{Abbreviations}

HOLA: Happy Older Latinos are Active

HIV: Human Immunodeficiency Virus

CHW: Community Health Worker

U.S.: United States

ART: Antiretroviral Therapy

MSM: Men Who Have Sex with Men

PWH: People With HIV

OAWH: Older Adults With HIV

LSMM: Latino Sexual Minority Men

SMM: Sexual Minority Men

MetS: Metabolic Syndrome

NIH: National Institutes of Health

IRB: Internal Review Board

SLT: Social Learning Theory

BA: Behavioral Activation

RA: Research Assistant

MMSE: Mini Mental Status Exam

$\mathrm{mL}$ : Milliliter

HDL-C: High-density lipoprotein cholesterol

LDL-C: Low-density lipoprotein cholesterol

HbA1c: Hemoglobin A1c

HOMA: Homeostatic Model Assessment 
SPSS: Statistical Package for the Social Sciences

ANOVA: Analysis Of Variance

\section{References}

1. CDC. HIV and Older Americans. Centers for Disease Control and Prevention. Published September 14, 2020. Accessed January $28,2021$. https://www.cdc.gov/hiv/group/age/olderamericans/index.html

2. CDC. HIV and Gay and Bisexual Men. Centers for Disease Control and Prevention. Published September 16, 2020. Accessed October 7, 2020. https://www.cdc.gov/hiv/group/msm/index.html

3. Karpiak SE, Lunievicz JL. Age Is Not a Condom: HIV and Sexual Health for Older Adults. Curr Sex Health Rep. 2017;9(3):109-115. doi:10.1007/s11930017-0119-0

4. 90-90-90: treatment for all | UNAIDS. Accessed January 27, 2021. https://www.unaids.org/en/resources/909090

5. Centers for Disease Control and Prevention. COVID-19 in Racial and Ethnic Minority Groups. Centers for Disease Control and Prevention. Published February 11, 2020. Accessed June 18, 2020. https://www.cdc.gov/coronavirus/2019-ncov/need-extra-precautions/racial-ethnic-minorities.html

6. U.S. Census Bureau. Hispanic or Latino Origin by Specific Origin.; 2018. Accessed April 28, 2020. https://data.census.gov/cedsci/table? q=hispanic\%20or\%20latino\%20miami\&g=0500000US12086\&hidePreview=true\&tid=ACSDT1Y2018.B03001\&t=Hispanic\%20or\%20Latino\&vintage=2018

7. Crepaz N, Tang T, Marks G, Hall HI. Viral Suppression Patterns Among Persons in the United States With Diagnosed HIV Infection in 2014. Ann Intern Med. 2017;167(6):446-447. doi:10.7326/L17-0278

8. Hood JE, Golden MR, Hughes JP, et al. Projected demographic composition of the United States population of people living with diagnosed HIV. AIDS Care. 2017;29(12):1543-1550. doi:10.1080/09540121.2017.1308466

9. Hoots BE, Finlayson TJ, Wejnert C, Paz-Bailey G. Updated Data on Linkage to Human Immunodeficiency Virus Care and Antiretroviral Treatment Among Men Who Have Sex With Men-20 Cities, United States. J Infect Dis. 2017;216(7):808-812. doi:10.1093/infdis/jix007

10. Linley L, Prejean J, An Q, Chen M, Hall HI. Racial/Ethnic Disparities in HIV Diagnoses Among Persons Aged 50 Years and Older in 37 US States, 20052008. Am J Public Health. 2012;102(8):1527-1534. doi:10.2105/AJPH.2011.300431

11. Cruz JJD, Karpiak SE, Brennan-Ing M. Health Outcomes for Older Hispanics With HIV in New York City Using the Oaxaca Decomposition Approach. Glob J Health Sci. 2015;7(1):133-143. doi:10.5539/gjhs.v7n1p133

12. Mindt MR, Miranda C, Arentoft A, et al. Aging and HIV/AIDS: Neurocognitive Implications for Older HIV-Positive Latina/o Adults. Behavioral Medicine. 2014;40(3):116-123. doi:10.1080/08964289.2014.914464

13. Marquine MJ, Heaton A, Johnson N, et al. Differences in Neurocognitive Impairment Among HIV-Infected Latinos in the United States. $J$ Int Neuropsychol Soc. 2018;24(2):163-175. doi:10.1017/S1355617717000832

14. Guaraldi G, Zona S, Alexopoulos N, et al. Coronary Aging in HIV-Infected Patients. Clin Infect Dis. 2009;49(11):1756-1762. doi:10.1086/648080

15. Kalayjian RC, Landay A, Pollard RB, et al. Age-related immune dysfunction in health and in human immunodeficiency virus (HIV) disease: association of age and HIV infection with naive CD8+ cell depletion, reduced expression of CD28 on CD8+ cells, and reduced thymic volumes.

16. Brennan, M., Karpiak, S.E., Shippy, R.A., \& Cantor, M.H. Older adults with HIV: An in-depth examination of an emerging population. 2009. New York: Nova Science.

17. Havlik RJ, Brennan M, Karpiak SE. Comorbidities and depression in older adults with HIV. Sex Health. 2011;8(4):551-559. doi:10.1071/SH11017

18. Frontini M, Chotalia J, Spizale L, Onya W, Ruiz M, Clark RA. Sex and Race Effects on Risk for Selected Outcomes among Elderly HIV-Infected Patients. Journal of the International Association of Physicians in AIDS Care. 2012;11(1):12-15. doi:10.1177/1545109711404947

19. Lombo B, Alkhalil I, Golden MP, et al. Prevalence of Metabolic Syndrome in Patients with HIV in the Era of Highly Active Antiretroviral Therapy. Conn Med. 2015;79(5):277-281.

20. Ramírez-Marrero FA, De Jesús E, Santana-Bagur J, Hunter R, Frontera W, Joyner MJ. Prevalence of Cardio-metabolic Risk Factors in Hispanics Living with HIV. Ethn Dis. 2010;20(4):423-428. Accessed July 10, 2020. https://www.ncbi.nlm.nih.gov/pmc/articles/PMC3071519/

21. Ramírez-Marrero FA, Rivera-Brown AM, Nazario CM, Rodríguez-Orengo JF, Smit E, Smith BA. Self-Reported Physical Activity in Hispanic Adults Living With HIV: Comparison With Accelerometer and Pedometer. Journal of the Association of Nurses in AIDS Care. 2008;19(4):283-294. doi:10.1016/j.jana.2008.04.003

22. Markides KS, Coreil J. The health of Hispanics in the southwestern United States: an epidemiologic paradox. Public Health Rep. 1986;101(3):253-265. Accessed July 10, 2020. https://www.ncbi.nlm.nih.gov/pmc/articles/PMC1477704/

23. Guaraldi G, Squillace N, Stentarelli C, et al. Nonalcoholic Fatty Liver Disease in HIV-Infected Patients Referred to a Metabolic Clinic: Prevalence, Characteristics, and Predictors. Clin Infect Dis. 2008;47(2):250-257. doi:10.1086/589294

24. Merriman RB. Nonalcoholic fatty liver disease and HIV infection. Curr HIV/AIDS Rep. 2006;3(3):113-117. doi:10.1007/BF02696654

25. 25.Aouizerat BE, Miaskowski CA, Gay C, et al. Risk Factors and Symptoms Associated With Pain in HIV-Infected Adults. Journal of the Association of Nurses in AIDS Care. 2010;21(2):125-133. doi:10.1016/j.jana.2009.10.003

26. Grov C, Golub SA, Parsons JT, Brennan M, Karpiak SE. Loneliness and HIV-related stigma explain depression among older HIV-positive adults. AIDS Care. 2010;22(5):630-639. doi:10.1080/09540120903280901

Page $8 / 11$ 
27. Horberg MA, Silverberg MJ, Hurley LB, et al. Effects of Depression and Selective Serotonin Reuptake Inhibitor Use on Adherence to Highly Active Antiretroviral Therapy and on Clinical Outcomes in HIV-Infected Patients. JAIDS Journal of Acquired Immune Deficiency Syndromes. 2008;47(3):384390. doi:10.1097/QAl.0b013e318160d53e

28. Hasin DS, Goodwin RD, Stinson FS, Grant BF. Epidemiology of major depressive disorder: results from the National Epidemiologic Survey on Alcoholism and Related Conditions. Arch Gen Psychiatry. 2005;62(10):1097-1106. doi:10.1001/archpsyc.62.10.1097

29. Landeiro F, Leal J, Gray AM. The impact of social isolation on delayed hospital discharges of older hip fracture patients and associated costs. Osteoporosis international: a journal established as result of cooperation between the European Foundation for Osteoporosis and the National Osteoporosis Foundation of the USA. 2016;27(2). Accessed January 28, 2021. https://ora.ox.ac.uk/objects/uuid:77d7ac37-b6a1-498d-836847d9ea3c5fe0

30. Brennan-Ing M, Seidel L, Karpiak SE. Social Support Systems and Social Network Characteristics of Older Adults with HIV. HIV and Aging. 2017;42:159172. doi:10.1159/000448561

31. Webel AR, Longenecker CT, Gripshover B, Hanson JE, Schmotzer BJ, Salata RA. Age, stress, and isolation in older adults living with HIV. AIDS Care. 2014;26(5):523-531. doi:10.1080/09540121.2013.845288

32. Dykstra PA, van Tilburg TG, Gierveld J de J. Changes in Older Adult Loneliness: Results From a Seven-Year Longitudinal Study. Res Aging. 2005;27(6):725-747. doi:10.1177/0164027505279712

33. Cornwell EY, Waite LJ. Measuring Social Isolation Among Older Adults Using Multiple Indicators From the NSHAP Study. J Gerontol B Psychol Sci Soc Sci. 2009;64B(suppl_1):i38-i46. doi:10.1093/geronb/gbp037

34. Luanaigh CO, Lawlor BA. Loneliness and the health of older people. Int J Geriatr Psychiatry. 2008;23(12):1213-1221. doi:10.1002/gps.2054

35. Holt-Lunstad J, Smith TB, Layton JB. Social relationships and mortality risk: a meta-analytic review. PLoS Med. 2010;7(7):e1000316. doi:10.1371/journal.pmed.1000316

36. J Infect Dis. 2003;187(12):1924-1933. doi:10.1086/375372

37. Vanable PA, Carey MP, Blair DC, Littlewood RA. Impact of HIV-Related Stigma on Health Behaviors and Psychological Adjustment Among HIV-Positive Men and Women. AIDS Behav. 2006;10(5):473-482. doi:10.1007/s10461-006-9099-1

38. Wilson DM, Errasti-Ibarrondo B, Low G. Where are we now in relation to determining the prevalence of ageism in this era of escalating population ageing? Ageing Research Reviews. 2019;51:78-84. doi:10.1016/j.arr.2019.03.001

39. Emlet CA. "You're Awfully Old to Have This Disease": Experiences of Stigma and Ageism in Adults 50 Years and Older Living With HIV/AIDS. The Gerontologist. 2006;46(6):781-790. doi:10.1093/geront/46.6.781

40. Yoo-Jeong M, Hepburn K, Holstad M, Haardörfer R, Waldrop-Valverde D. Correlates of loneliness in older persons living with HIV. AIDS Care. 2019;0(0):18. doi:10.1080/09540121.2019.1659919

41. Miyawaki CE. Association of social isolation and health across different racial and ethnic groups of older Americans. Ageing Soc. 2015;35(10):22012228. doi:10.1017/S0144686X14000890

42. Tomaka J, Thompson S, Palacios R. The Relation of Social Isolation, Loneliness, and Social Support to Disease Outcomes Among the Elderly. J Aging Health. 2006;18(3):359-384. doi:10.1177/0898264305280993

43. Kraemer HC, Mintz J, Noda A, Tinklenberg J, Yesavage JA. Caution Regarding the Use of Pilot Studies to Guide Power Calculations for Study Proposals. Archives of General Psychiatry. 2006;63(5):484-489. doi:10.1001/archpsyc.63.5.484

44. Jimenez DE, Reynolds CF, Alegría M, Harvey P, Bartels SJ. The Happy Older Latinos are Active (HOLA) health promotion and prevention study: study protocol for a pilot randomized controlled trial. Trials. 2015;16(1):579. doi:10.1186/s13063-015-1113-3

45. Heckman TG, Sikkema KJ, Hansen N, et al. A randomized clinical trial of a coping improvement group intervention for HIV-infected older adults. Journal of Behavioral Medicine. 2011;2(34):102-111. doi:10.1007/s10865-010-9292-6

46. Rissel C, Passmore E, Mason C, Merom D. Two Pilot Studies of the Effect of Bicycling on Balance and Leg Strength among Older Adults. Journal of Environmental and Public Health. doi:https://doi.org/10.1155/2013/686412

47. Bandura A. Social Learning Theory. Prentice-Hall; 1977.

48. Jacobson NS, Martell CR, Dimidjian S. Behavioral activation treatment for depression: Returning to contextual roots. Clinical Psychology: Science and Practice. 2001;8(3):255-270. doi:10.1093/clipsy.8.3.255

49. Perez M, Findley SE, Mejia M, Martinez J. The Impact of Community Health Worker Training and Programs in NYC. Journal of Health Care for the Poor and Underserved. 2006;17(1):26-43. doi:10.1353/hpu.2006.0049

50. Patel AR, Nowalk MP. Expanding immunization coverage in rural India: A review of evidence for the role of community health workers. Vaccine. 2010;28(3):604-613. doi:10.1016/j.vaccine.2009.10.108

51. Leveille SG, Wagner EH, Davis C, et al. Preventing Disability and Managing Chronic Illness in Frail Older Adults: A Randomized Trial of a CommunityBased Partnership with Primary Care. Journal of the American Geriatrics Society. 1998;46(10):1191-1198. doi:https://doi.org/10.1111/j.15325415.1998.tb04533.x

52. Prochaska JO, DiClemente CC. Transtheoretical therapy: Toward a more integrative model of change. Psychotherapy: Theory, Research \& Practice. 1982;19(3):276-288. doi:10.1037/h0088437

53. Reynolds CF, Thomas SB, Morse JQ, et al. Early Intervention to Preempt Major Depression Among Older Black and White Adults. PS. 2014;65(6):765-773. doi:10.1176/appi.ps.201300216

Page $9 / 11$ 
54. Whyte EM, Rovner B. Depression in late-life: shifting the paradigm from treatment to prevention. International Journal of Geriatric Psychiatry. 2006;21(8):746-751. doi:https://doi.org/10.1002/gps.1555

55. Jimenez DE, Cook B, Kim G, et al. Assessing the Relationship between Physical Illness and Mental Health Service Use and Expenditures among Older Adults from Racial/Ethnic Minority Groups. Psychiatr Serv. 2015;66(7):727-733. doi:10.1176/appi.ps.201400246

56. Jimenez D, Reynolds CF, Alegria M, Harvey PS, Bartels SJ. Preventing Anxiety in Depression in Older Latinos: The Happy Older Latinos are Active (HOLA) Health Promotion Study. The American Journal of Geriatric Psychiatry. 2017;25(3):S124. doi:10.1016/j.jagp.2017.01.156

57. Folstein MF, Folstein SE, McHugh PR. Mini-mental state: A practical method for grading the cognitive state of patients for the clinician. Journal of Psychiatric Research. 1975;12(3):189-198. doi:10.1016/0022-3956(75)90026-6

58. Peters DM, Fritz SL, Krotish DE. Assessing the reliability and validity of a shorter walk test compared with the 10-Meter Walk Test for measurements of gait speed in healthy, older adults. J Geriatr Phys Ther. 2013;36(1):24-30. doi:10.1519/JPT.0b013e318248e20d

59. About \#UequalsU | United States | Prevention Access Campaign. prevention. Accessed January 28, 2021. https://www.preventionaccess.org/about

60. Radloff LS. The CES-D Scale: A Self-Report Depression Scale for Research in the General Population. Applied Psychological Measurement. 1977;1(3):385-401. doi:10.1177/014662167700100306

61. Kroenke K, Spitzer RL, Williams JB. The Patient Health Questionnaire-2: validity of a two-item depression screener. Medical care. 2003;41(11):12841292.

62. Cohen S, Kamarck T, Mermelstein R. A Global Measure of Perceived Stress. Journal of Health and Social Behavior. 1983;24(4):385-396. doi:10.2307/2136404

63. Spitzer RL, Kroenke K, Williams JBW, Löwe B. A Brief Measure for Assessing Generalized Anxiety Disorder: The GAD-7. Arch Intern Med. 2006;166(10):1092-1097. doi:10.1001/archinte.166.10.1092

64. Zimet GD, Powell SS, Farley GK, Werkman S, Berkoff KA. Psychometric characteristics of the multidimensional scale of perceived social support. Journal of personality assessment. 1990;55(3-4):610-617. Accessed December 17, 2015.

http://www.tandfonline.com/doi/abs/10.1080/00223891.1990.9674095

65. Marin G, Gamba RJ. A New Measurement of Acculturation for Hispanics: The Bidimensional Acculturation Scale for Hispanics (BAS). Hispanic Journal of Behavioral Sciences. 1996;18(3):297-316. doi:10.1177/07399863960183002

66. Bull FC, Maslin TS, Armstrong T. Global Physical Activity Questionnaire (GPAQ): Nine Country Reliability and Validity Study. Journal of Physical Activity and Health. 2009;6(6):790-804. doi:10.1123/jpah.6.6.790

67. Ware J, Kosinski M, Keller SD. A 12-Item Short-Form Health Survey: construction of scales and preliminary tests of reliability and validity. Med Care. 1996;34(3):220-233. doi:10.1097/00005650-199603000-00003

68. Berger BE, Ferrans CE, Lashley FR. Measuring stigma in people with HIV: Psychometric assessment of the HIV stigma scale. Research in Nursing \& Health. 2001;24(6):518-529. doi:https://doi.org/10.1002/nur.10011

69. Susskind EC, Howland EW. Measuring Effect Magnitude in Repeated Measures ANOVA Designs: Implications for Gerontological Research. Journal of Gerontology. 1980;35(6):867-876. doi:10.1093/geronj/35.6.867

70. Conner KO, Copeland VC, Grote NK, et al. Mental Health Treatment Seeking Among Older Adults With Depression: The Impact of Stigma and Race. The American Journal of Geriatric Psychiatry. 2010;18(6):531-543. doi:10.1097/JGP.0b013e3181cc0366

71. Jimenez DE, Bartels SJ, Cardenas V, Alegría M. Stigmatizing attitudes toward mental illness among racial/ethnic older adults in primary care. International Journal of Geriatric Psychiatry. 2013;28(10):1061-1068. doi:https://doi.org/10.1002/gps.3928

72. Jimenez DE, Begley A, Bartels SJ, et al. Improving Health-Related Quality of Life in Older African American and Non-Latino White Patients. The American Journal of Geriatric Psychiatry. 2015;23(6):548-558. doi:10.1016/j.jagp.2014.08.001

73. Jimenez DE, Alegría M, Chen C, Chan D, Laderman M. Prevalence of Psychiatric Illnesses in Older Ethnic Minority Adults. Journal of the American Geriatrics Society. 2010;58(2):256-264. doi:https://doi.org/10.1111/j.1532-5415.2009.02685.x

74. Kenya S, Jones J, Arheart K, et al. Using Community Health Workers to Improve Clinical Outcomes Among People Living with HIV: A Randomized Controlled Trial. AIDS Behav. 2013;17(9):2927-2934. doi:10.1007/s10461-013-0440-1

75. Barbour KA, Blumenthal JA. Exercise training and depression in older adults. Neurobiology of Aging. 2005;26(1, Supplement):119-123. doi:10.1016/j.neurobiolaging.2005.09.007

76. Martinson BC, Crain AL, Sherwood NE, Hayes M, Pronk NP, O'Connor PJ. Maintaining physical activity among older adults: Six-month outcomes of the Keep Active Minnesota randomized controlled trial. Preventive Medicine. 2008;46(2):111-119. doi:10.1016/j.ypmed.2007.08.007

77. Tobias CR, Cunningham W, Cabral HD, et al. Living with HIV But Without Medical Care: Barriers to Engagement. AIDS Patient Care and STDs. 2007;21(6):426-434. doi:10.1089/apc.2006.0138

78. Warren-Jeanpiere L, Dillaway H, Hamilton P, Young M, Goparaju L. Taking It One Day at a Time: African American Women Aging with HIV and CoMorbidities. AIDS Patient Care and STDs. 2014;28(7):372-380. doi:10.1089/apc.2014.0024

79. Sangaramoorthy T, Jamison AM, Dyer TV. HIV Stigma, Retention in Care, and Adherence Among Older Black Women Living With HIV. Journal of the Association of Nurses in AIDS Care. 2017;28(4):518-531. doi:10.1016/j.jana.2017.03.003

\section{Tables}

Table 1. Inclusion/Exclusion Criteria 


\begin{tabular}{|c|c|}
\hline $\begin{array}{l}\text { Inclusion } \\
\text { Criteria }\end{array}$ & Exclusion Criteria \\
\hline $\begin{array}{l}\text { Latino (self- } \\
\text { identified) }\end{array}$ & Have a diabetes diagnosis \\
\hline Age 50+ & $\begin{array}{l}\text { Have a diagnosis of any neurodegenerative disorder or dementia (Parkinson's disease, Alzheimer's, vascular, } \\
\text { frontotemporal dementia, etc.) or significant cognitive impairment as indicated by a Mini Mental Status Exam score } \\
<24\end{array}$ \\
\hline Male & Have contraindications to physical activity outlined in the American College of Sports Medicine standards \\
\hline $\begin{array}{l}\text { HIV infected } \\
\text { but are } \\
\text { virologically } \\
\text { suppressed } \\
\text { (viral load } \\
<200 \\
\text { copies } / \mathrm{mL} \text { ) }\end{array}$ & Are unable to complete $10 \mathrm{~m}$ walk test \\
\hline $\begin{array}{l}\text { Volunteer } \\
\text { informed } \\
\text { consent }\end{array}$ & Currently residing in a nursing or group home \\
\hline $\begin{array}{l}\text { Expect to stay } \\
\text { in Miami for the } \\
\text { next } 6 \text { months }\end{array}$ & Have a terminal physical illness expected to result in the death within one year \\
\hline $\begin{array}{l}\text { Have } \\
\text { documented } \\
\text { risk of } \\
\text { cardiometabolic } \\
\text { disease. }\end{array}$ & $\begin{array}{l}\text { Have an acute or severe medical illness that precludes them from safely participating in a health promotion } \\
\text { intervention (e.g. progressive, degenerative neurologic disease, such as Parkinson's Disease, multiple sclerosis, } \\
\text { ALS; severe arthritis or orthopedic condition that would prevent participation in a physical activity program; lung } \\
\text { disease requiring either oral or injected steroids, or the use of supplemental oxygen; New York Heart Association } \\
\text { Class III or IV congestive heart failure, clinically significant aortic stenosis, history of cardiac arrest, use of a } \\
\text { cardiac defibrillator, or uncontrolled angina; renal disease requiring the use of dialysis; cancer being actively } \\
\text { treated with radiation or chemotherapy; myocardial infarction, CABG, or valve replacement within the past } 6 \\
\text { months; serious conduction disorder, such as 3rd degree heart block; uncontrolled arrhythmia; pulmonary } \\
\text { embolism or deep venous thrombosis within past 6-months; uncontrolled diabetes with recent weight loss, diabetic } \\
\text { coma or frequent insulin reactions; stroke, hip fracture, hip or knee replacement, or spinal surgery in the past } 6 \\
\text { months; receiving physical therapy for gait, balance, or other lower extremity training; severe, uncontrolled } \\
\text { hypertension -systolic blood pressure >200 mmHg and/or diastolic blood pressure >110 mmHg) } \\
\text { Are currently taking antidepressant medications in doses indicated for weight reduction. }\end{array}$ \\
\hline & \\
\hline
\end{tabular}

\section{Figures}

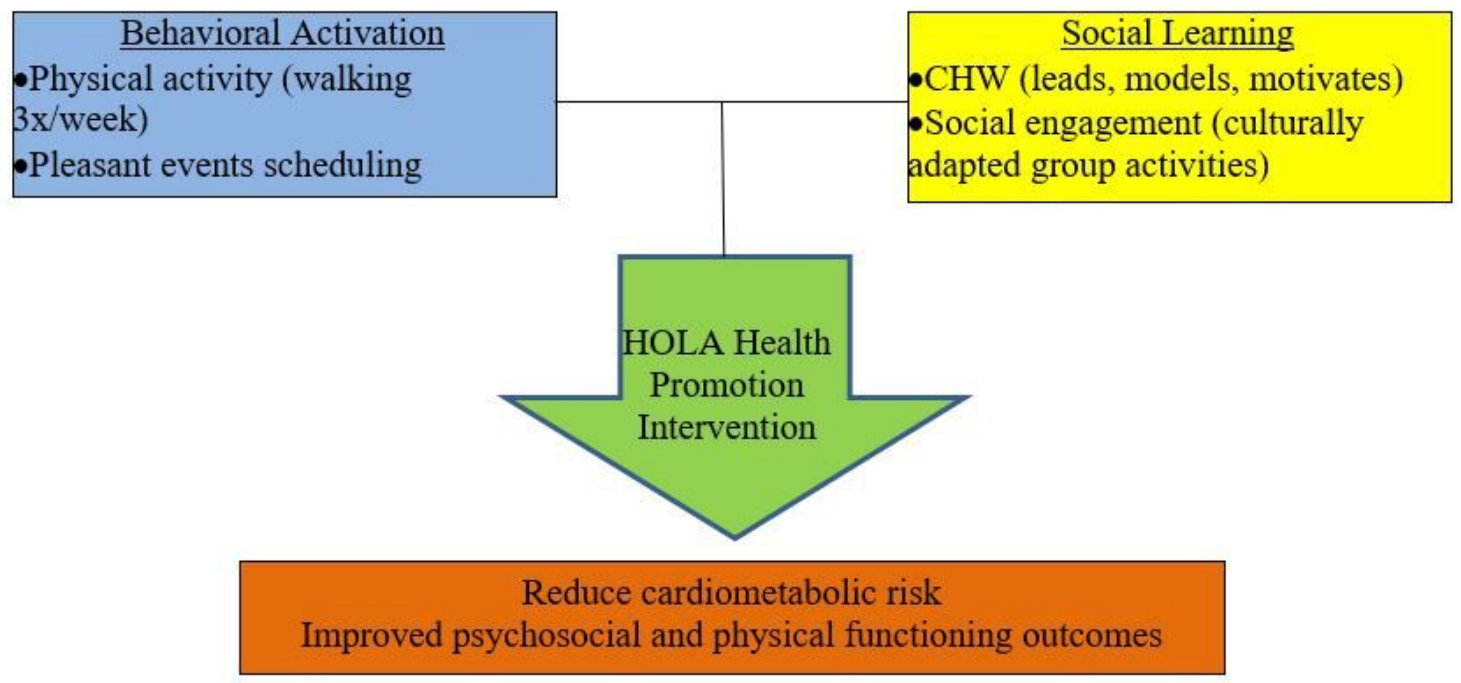

Figure 1

Conceptual Framework 